\title{
Design and experiments of a five-fold helically corrugated waveguide for microwave pulse compression
}

\author{
Liang Zhang ${ }^{1}$, Sergey V. Mishakin ${ }^{2}$, Wenlong He ${ }^{1}$, Sergey V. Samsonov ${ }^{2}$, Adrian W. Cross ${ }^{1}$, \\ Gregory G. Denisov ${ }^{2}$, Michael McStravick ${ }^{1}$, Vladimir L. Bratman ${ }^{2}$, Colin G. Whyte ${ }^{1}$, Craig W. Robertson ${ }^{1}$, \\ Alan R. Young ${ }^{1}$, Huabi Yin ${ }^{1}$, Kevin Ronald ${ }^{1}$, Philip MacInnes ${ }^{1}$ and Alan D.R. Phelps ${ }^{1}$ \\ ${ }^{1}$ Department of Physics, SUPA, University of Strathclyde, Glasgow, G4 0NG, UK \\ ${ }^{2}$ Institute of Applied Physics, Russian Academy of Sciences, Nizhny Novgorod, 603950, Russia
}

\begin{abstract}
Metal waveguide can be used as a dispersive medium to convert long duration, lower power pulses into short, higher peak power pulses. This provides an advanced method to generate radiation with gigawatts power in the millimeter and sub-millimeter wavelength range by compressing a megawatt level long duration pulse. In this paper, a five-fold helically corrugated waveguide operating in $\mathrm{X}$-band was designed and constructed. The experiments conducted show that a $5.75 \mathrm{~kW}$ average power microwave pulse with a $6 \%$ bandwidth and duration of $80 \mathrm{~ns}$ can be compressed into a $144.8 \mathrm{~kW}, 1.6 \mathrm{~ns}$ pulse with a power compression factor of 25.2 .
\end{abstract}

\section{INTRODUCTION}

$\mathrm{P}$ ulse compression technology can be used in applications that require high peak power and pulsed operation, such as radar systems to improve the space and time resolution, and the generation of intense ultra-short laser pulses. The use of a metal waveguide as a dispersive medium to generate high-peak-power microwaves from low-power pulses was proposed in [1]. The principle of the pulse compression process is to use a frequency modulated pulse traveling through a dispersive media in which different frequency components have different group velocities. If the group velocities of the pulse train are monotonously increasing versus the entrance time, the pulse width will reduce and the peak power will increase. The maximum compression ratio happens at the exit of the waveguide where all the frequency components arrive at the same time.

The pulse compressor requires a dispersion curve with a monotonic group velocity and low losses in a reasonable wide frequency band. It can be met by the helically corrugated waveguide (HCW) with both axial and azimuthal periodicity that couples modes in the circular waveguide to generate new operating eigenwaves, as shown in Fig. 1.

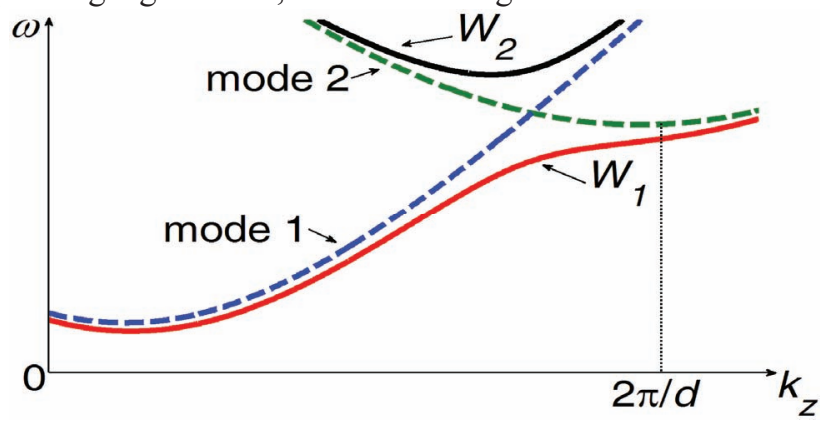

Fig. 1. The dispersion curve of the HCW.

The operating mode $W_{1}$ has a monotonically decreasing group velocity over a wide frequency band around the coupling point and also a low Ohmic loss as it is far from the cutoff. The HCW has successfully been used in the gyro-TWA
[2] and gyro-BWO [3]. The first pulse compression experiment using the $\mathrm{HCW}$ was carried out on a three-fold waveguide, in which the operating mode was obtained by coupling the spatial harmonic $\mathrm{TE}_{11}$ mode and the $\mathrm{TE}_{21}$ mode. A peak power compression ratio of 11 was achieved [1]. Further research was carried out to optimize the frequency sweep for the three-fold compressor resulting in an increase in the power compression factor to 25 [4]. To achieve higher power capability, a pulse compressor with larger dimensions using a five-fold $\mathrm{HCW}$, in which the operating mode is obtained by coupling the spatial harmonic $\mathrm{TE}_{31}$ and the $\mathrm{TE}_{22}$ modes was designed and constructed $[5,6]$.

\section{DESIGN OF THE HELICALLY CORRUGATED WAVEGUIDE}

The HCW was designed to have a mean waveguide diameter at least twice that of the three-fold one while operating in the same frequency range. The dispersion characteristic is totally determined by the HCW's geometry whose profile is governed by the expression $r(\theta, z)=R_{0}+R_{l} \cos (5 \theta+2 \pi z / d)$ in a cylindrical coordinate system $(r, \theta, z)$. The mean radius $R_{0}$ mainly decides the operating frequency range; the corrugation depth $R_{l}$ mostly controls the coupling strength, and the period $d$ directly affects the position of the coupling point. Different methods have been developed to calculate the dispersion curve with different accuracies and computing times, including 1D coupled wave theory based on the method of perturbation [7], the 2D finite element method (FEM) eigensolver based on helicoidal coordinate transform [8], and the full 3D FDTD transient solver, or the FEM eigensolver [9]. The optimal dimensions used in the experiments were derived to be a five-fold HCW with $R_{0}=32.84 \mathrm{~mm}, R_{1}=2.43 \mathrm{~mm}$, and $d=33.26 \mathrm{~mm}$. The total length of $2.86 \mathrm{~m}$ was decided by the optimum trade-off between compression ratio and the energy efficiency. The simulated dispersion curves obtained by the different methods and the experimental measurement are shown in Fig. 2.

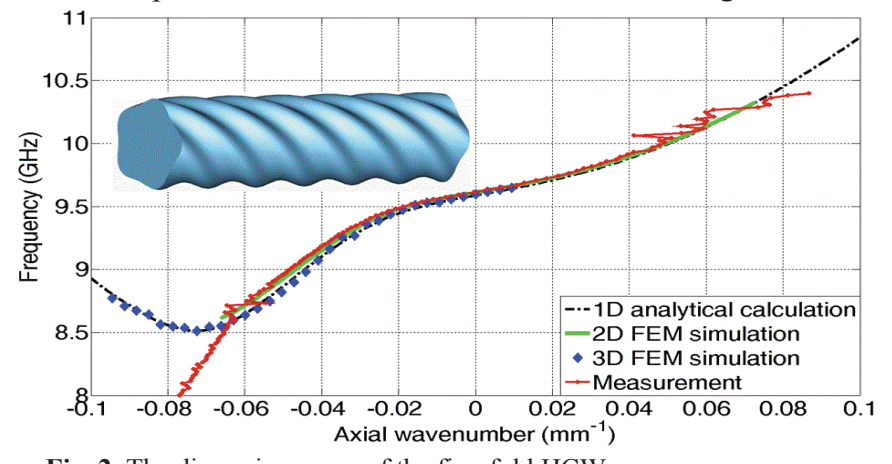

Fig. 2. The dispersion curve of the five-fold HCW. 
The 3D FDTD simulation code CST Microwave Studio was used to study the dynamic electric and magnetic fields inside the HCW. It was found that the maximum power capability of the five-fold $\mathrm{HCW}$ is about 7 times higher as compared with the three-fold one operating in the same frequency range.

To excite the circularly polarized $\mathrm{TE}_{31}$ mode in the five-fold $\mathrm{HCW}$, a mode convertor was designed to transfer the input $\mathrm{TE}_{11}$ mode into the $\mathrm{TE}_{31}$ mode. The mode conversion efficiency was found to be more than $95 \%$ in the frequency range of $8.9-9.9 \mathrm{GHz}$ and the reflection coefficient of the $\mathrm{TE}_{11}$ mode is less than $-30 \mathrm{~dB}$. As the four-fold mode convertor requires a circularly polarized $\mathrm{TE}_{11}$ wave as the input, an elliptical polarizer was designed and constructed that was able to convert the polarization of the $\mathrm{TE}_{11}$ wave between linear polarization and circular polarization. The center frequency was set at $9.3 \mathrm{GHz}$ and it was able to achieve more than $98 \%$ energy conversion from one polarization to the other. The connection of the waveguide structure is shown in Fig. 3. At the other end of the five-fold HCW the same configuration was used.

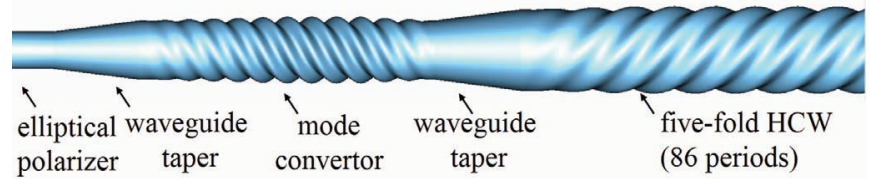

Fig. 3. The structure of the pulse compressor.

\section{EXPERIMENT SETUP AND RESULTS}

The five-fold $\mathrm{HCW}$ and the four-fold mode convertor were constructed separately. The inner surface of the HCW and the mode convertors were formed by machining at Strathclyde positive aluminium mandrels using a 4-axis computer numerical control $(\mathrm{CNC})$ machine with the copper grown on the aluminum by electroforming with a thickness of 6 millimeters. Finally the aluminum was chemically dissolved to leave the copper waveguides. The waveguides were then carefully joined and tightened together.

The pulse compression experiment was carried out using the designed HCW and the schematic of the experimental setup is shown in Fig. 4. An Agilent N6030A arbitrary waveform generator (AWG) was programmed to generate the input frequency sweeping signal (about $0.6 \mathrm{GHz}$ bandwidth). The signal was then sent to the mixers of an Agilent E8257D vector signal generator (VSG) to generate the input signal in the frequency range from $9.65 \mathrm{GHz}$ to $9.05 \mathrm{GHz}$. A conventional X-band TWT was used to amplify the input signal up to a few $\mathrm{kW}$. The input and output compressed pulses were recorded by a $20 \mathrm{GHz}$ Agilent DSOX92004A real time digitizing storage oscilloscope.

In the experiments, with the optimal frequency sweep, a maximum peak power compression ratio of 25.2 was achieved by compressing a $80 \mathrm{~ns} 5.75 \mathrm{~kW}$ pulse to a $1.6 \mathrm{~ns} 144.8 \mathrm{~kW}$ pulse, as shown in Fig. 5.

A five-fold helically corrugated compressor was used as the dispersive medium where a $700 \mathrm{MW}$ X-band relativistic backward wave oscillator (RBWO) served as a 20ns duration source of the frequency modulated pulses. Compression of pulses down to a halfwidth of $2.2 \mathrm{~ns}$ accompanied by a 4.5 -fold power increase up to a value of $\sim 3.2 \mathrm{GW}$ was achieved [10]. Results have been presented demonstrating that it may be possible to use a 5-fold helically corrugated waveguide compressor combined with a RBWO to produce peak powers in excess of $10 \mathrm{GW}$ in X-band.

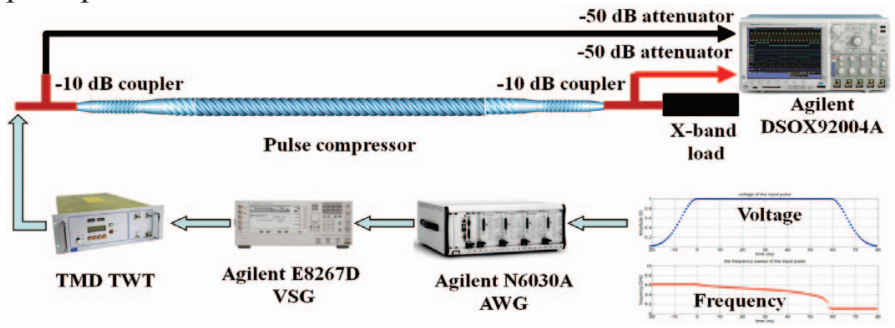

Fig. 4. The schematic of the microwave pulse compression setup.

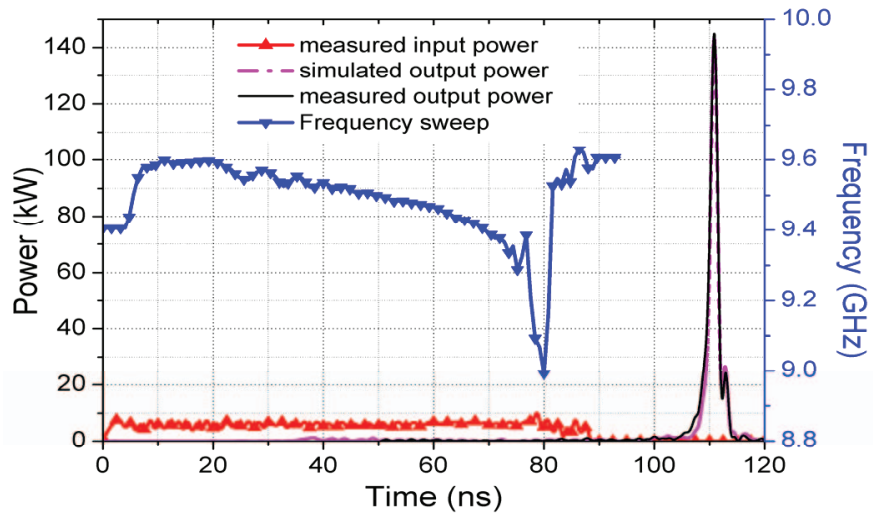

Fig. 5. The measured and simulated microwave outputs from the compression experiment and input microwave waveform and frequency sweep.

\section{REFERENCES}

[1] S. V. Samsonov, A. D. R. Phelps, V .L. Bratman, et al., "Compression of frequency modulated pulses using helically corrugated waveguides and its potential for generating multigigawatt rf radiation," Phys. Rev. Lett., vol. 92, no. 11, 118301, Mar. 2004.

[2] G. G. Denisov, V. L. Bratman, A. W. Cross, et al, "Gyrotron travelling wave amplifier with a helical interaction waveguide," Phys. Rev. Lett., vol. 81, no. 25, pp. 5680-5683, Dec. 1998.

[3] W. He, C. R. Donaldson, L. Zhang, et al., "High Power Wideband Gyrotron Backward Wave Oscillator Operating towards the Terahertz Region," Phys. Rev. Lett., vol. 110, pp. 165101, Apr. 2013.

[4] M. McStravick, S. V. Samsonov, K. Ronald, et al., "Experimental results on microwave pulse compression using helically corrugated waveguide," J. Appl. Phys., vol. 108, no. 5, 054908, Sept. 2010.

[5] L. Zhang, W. He, K. Ronald, et al., "Multi-mode coupling wave theory for helically corrugated waveguide," IEEE Trans. Microw. Theory Techn., vol. 60, no. 1, pp. 1-7, Jan. 2012.

[6] L. Zhang, S. V. Mishakin, W. He, et al., "Experimental study of microwave pulse compression using a five-fold helically corrugated waveguide", IEEE Trans. Microw. Theory Techn., vol. 63, no. 3, pp. 1090-1096, Mar. 2015.

[7] G. G. Denisov and M. G. Reznikov, "Corrugated Cylindrical Resonators for Short-wavelength Relativistic Microwave Oscillators," Radiophysics and Quantum Electronics, vol. 25, no. 5, pp. 407-413, May 1982.

[8] S. V. Mishakin and S. V. Samsonov, "Analysis of Dispersion and Losses in Helically Corrugated Metallic Waveguides by 2-D Vector Finite-Element Method," IEEE Trans. Microw. Theory Techn., vol. 59, no. 9, pp. 2189-2196, Sept. 2011.

[9] G. Burt, S. V. Samsonov, K. Ronald, et al., "Dispersion of helically corrugated waveguides: Analytical, numerical, and experimental study," Phys. Rev. E, vol. 70, pp. 046402, Oct. 2004.

[10] V. L. Bratman, G. G. Denisov, N. G. Kolganov, et al., "Generation of 3 $\mathrm{GW}$ microwave pulses in X-band from a combination of a relativistic backward-wave oscillator and a helical-waveguide compressor," Phys. Plasmas, vol. 17, pp. 110703, Nov. 2010. 